\title{
A Three-Parameter Non-linear Lattice-Boltzmann Model for Ideal Miscible Fluids
}

\author{
Paulo Cesar Facin ${ }^{1}$, Paulo Cesar Philippi ${ }^{2}$, and \\ Luís Orlando Emerich dos Santos ${ }^{2}$ \\ 1 Physics Department, State University of Ponta Grossa \\ 84030-900 Ponta Grossa, PR, Brazil \\ facin@lmpt.ufsc.br \\ 2 Mechanical Engineering Department, Federal University of Santa Catarina, \\ 88040-900, Florianópolis, SC, Brazil \\ \{philippi, emerich\}@lmpt.ufsc.br
}

\begin{abstract}
Present work is concerned with the construction of a latticeBoltzmann (LB) model for ideal miscible fluids. In this particular case, collision term in LB equation can be modelled by, only, considering mutual and cross collisions between, respectively, particles of the same and of different kind. A non-linear LB model with three distinct relaxation times intended to be used in problems with large concentration gradients is presented. Model enables the independent management of the fluid viscosities $\mu^{r}$ and $\mu^{b}$ and binary diffusivity $\mathcal{D}$. It is shown that mass and momentum are, always, preserved and that the model retrieves consistent hydrodynamic equations in the incompressible limit. Theoretical values, obtained from Chapman-Enskog analysis, for binary diffusivity and mixture viscosity are compared with numerical values, directly obtained from LB simulations.
\end{abstract}

\section{Introduction}

When two fluids $r$ and $b$ are mixed, long-range intermolecular forces will control the nature of the resulting physical system. When attractive $r-r$ and $b-b$ forces are dominant with respect to $r-b$ forces, fluids $\mathrm{r}$ and $\mathrm{b}$ will segregate. In this case, fluids are said to be immiscible. Mixtures of fluids can be thermodynamically stable when long-range attraction between molecules of different kinds are dominant. In this second case, fluids are said to be miscible. When long-range forces are negligeable, the fluids and their mixtures are said to be ideal.

A review on lattice-Boltzmann models (LB) for miscible fluids has been, recently, given by Sofonea and Sekerka[1]. Most common LB are based on a single BGK collision term and momentum conservation requires the relaxation times for the species $k$ to be identical [2],[3],[4]. In this way, simulation is restricted to fluids with identical viscosity. Sofonea and Sekerka proposed the following splitting of the collision term,

$$
\Omega_{i}^{r}=\left(\frac{\rho^{r}}{\tau^{r}}+\frac{\rho^{b}}{\tau^{r b}}\right)\left[R_{i}^{e q}\left(\rho^{r}, \boldsymbol{u}\right)-R_{i}\right],
$$




$$
\Omega_{i}^{b}=\left(\frac{\rho^{b}}{\tau^{b}}+\frac{\rho^{r}}{\tau^{b r}}\right)\left[B_{i}^{e q}\left(\rho^{b}, \boldsymbol{u}\right)-B_{i}\right],
$$

where, for fluids $r$ and $b, \rho^{r}, \rho^{b}$ are the densities, the $\tau^{\prime}$ 's are relaxation times, $R_{i}, B_{i}$ are the distribution functions, and $R_{i}^{e q}, B_{i}^{e q}$ denotes the equilibrium distributions, calculated using the mixture velocity $\boldsymbol{u}$.

Momentum preservation requires

$$
\left(\frac{\rho^{r}}{\tau^{r}}+\frac{\rho^{b}}{\tau^{r b}}\right)=\left(\frac{\rho^{b}}{\tau^{b}}+\frac{\rho^{r}}{\tau^{b r}}\right)=\frac{1}{\tau},
$$

or $\tau^{b r}=\tau^{r}$ and $\tau^{r b}=\tau^{b}$. In this way, Sofonea and Sekerka's model use two independent relaxation times, replacing the constant relaxation time by a function of the fractional mass of $\mathrm{r}$ and $\mathrm{b}$ particles.

In this work, a three-parameter BGK model is presented for ideal miscible fluids, enabling the independent management of the mass diffusivity and $r$ and $b$ fluid viscosities.

Although in a different form, a three-parameter model with the same above features has been, very recently, proposed by Luo and Girimaji[5]. Luo and Gimaji's rapid communication was published in the course of present work and, in spite of the fact that a detailed comparison work is needed considering the computacional performance of the two models, some comments are given in the Conclusions section, for clarifying some main differences that could be important.

\section{Model}

Considering $R_{i}(\boldsymbol{X}, T)$ to be the particles distribution of $r$-particles in site $\boldsymbol{X}$ at time $T$ and, similarly, for $B_{i}(\boldsymbol{X}, T)$, lattice-Boltzmann equation for kind $K$ particles, $K=R, B$, will be written as

$$
K_{i}\left(\boldsymbol{X}+\boldsymbol{c}_{i}, T+1\right)=K_{i}(\boldsymbol{X}, T)+\Omega_{i}^{k}\left(R_{0}, R_{1}, \ldots, R_{b_{m}}, B_{0}, B_{1}, \ldots, B_{b_{m}}\right),
$$

where $\boldsymbol{c}_{i}=$ lattice unitary velocity along direction $i, b_{m}=$ number of lattice directions and index 0 corresponds to rest particles. Collision operator $\Omega_{i}^{k}, k=$ $r, b$ is required to satisfy mass and momentum conservation:

$$
\begin{gathered}
\sum_{i=0}^{b_{m}} \Omega_{i}^{r}=0, \\
\sum_{i=0}^{b_{m}} \Omega_{i}^{b}=0, \\
\sum_{i=0}^{b_{m}}\left(\Omega_{i}^{r}+\Omega_{i}^{b}\right) \boldsymbol{c}_{i}=0 .
\end{gathered}
$$


A three-parameter BGK collision term that satisfies the above restrictions is proposed in present work, written as

$$
\begin{aligned}
& \Omega_{i}^{r}=\omega^{r} \frac{R_{i}^{e q}\left(\rho^{r}, \boldsymbol{u}^{r}\right)-R_{i}}{\tau^{r}}+\omega^{b} \frac{R_{i}^{e q}\left(\rho^{r}, \boldsymbol{u}^{b}\right)-R_{i}}{\tau^{m}}, \\
& \Omega_{i}^{b}=\omega^{b} \frac{B_{i}^{e q}\left(\rho^{b}, \boldsymbol{u}^{b}\right)-B_{i}}{\tau^{b}}+\omega^{r} \frac{B_{i}^{e q}\left(\rho^{b}, \boldsymbol{u}^{r}\right)-B_{i}}{\tau^{m}},
\end{aligned}
$$

where

$$
\omega^{k}=\frac{\rho^{k}}{\rho}
$$

and

$$
\begin{gathered}
\rho^{k}=\sum_{i=0}^{b_{m}} K_{i}, \\
\boldsymbol{u}^{k}=\frac{1}{\rho^{k}} \sum_{i=0}^{b_{m}} K_{i} \boldsymbol{c}_{i},
\end{gathered}
$$

are, respectively, the macroscopic density and velocity of component $k, k=r, b$. Equilibrium distributions are taken from the single-fluid $\mathcal{O}\left(v^{2}\right)$ lattice-Boltzmann equilibrium distributions as[6],

$$
\begin{gathered}
K_{i}^{0}\left(\rho^{k}, \boldsymbol{v}\right)=\frac{\rho^{k}}{b}+\frac{D \rho^{k}}{b_{m} c^{2}} c_{i \alpha} v_{\alpha} \\
+\frac{D(D+2) \rho^{k}}{2 b_{m} c^{4}} c_{i \alpha} c_{i \beta} v_{\alpha} v_{\beta}-\frac{D \rho^{k}}{2 b_{m} c^{2}}(v)^{2}, i=1 \ldots b_{m} \\
K_{0}^{0}\left(\rho^{k}, \boldsymbol{v}\right)=\frac{\rho^{k}}{b} b_{r}-\frac{\rho^{k}}{c^{2}}(v)^{2}
\end{gathered}
$$

for a generic velocity $\boldsymbol{v}$ and $k=r, b$. In the above equation, as usually, $D$ is the lattice Euclidean dimension, $c=\left|\boldsymbol{c}_{i}\right|, b_{r}$ is a free-parameter related to the distribution of rest particles, $b=b_{r}+b_{m}$. The first term in the r.h.s. of Eq. (8) is related to the relaxation of $r$-particles distribution to an equilibrium state given by $r$-component density and velocity, considering $r-r$ collisions, only. The second term considers $r$ - $b$ collisions and is related to the relaxation of $r$-particles to an equilibrium state given by density $\rho^{r}$ and by a $b$ velocity $\boldsymbol{u}^{b}$. When component $b$ is dominant, i.e., when $\rho^{b} / \rho \rightarrow 1$, collisions $r-r$ are very infrequent and collisions $r$ - $b$ will impose velocity $\boldsymbol{u}^{b}$ to component $r$. 


\section{Chapman-Enskog Asymptotic Analysis}

In the continuum limit, the Knudsen number $\mathcal{K}_{n}=h / L \rightarrow 0$, where $h$ is the length corresponding to a lattice unit and $L$ is a macroscopic length, distribution $K_{i}(\boldsymbol{X}, T)$ is expanded in powers of $\mathcal{K}_{n}$,

$$
K_{i}=K_{i}^{0}+\mathcal{K}_{n} K_{i}^{1}+\ldots, \quad K=R, B ; \quad i=0,1, \ldots, b_{m},
$$

and the time derivative has an induced decomposition

$$
\partial_{t}=\partial_{0}+\mathcal{K}_{n} \partial_{1}+\ldots
$$

Collision term given by Eq. (8) can be expanded around $R_{i}^{e q}\left(\rho^{r}, \boldsymbol{u}\right), \boldsymbol{u}=\omega^{r} \boldsymbol{u}^{r}+$ $\omega^{b} \boldsymbol{u}^{b}$, giving

$$
\begin{aligned}
\Omega_{i}^{r} & =\left(\frac{\omega^{r}}{\tau^{r}}+\frac{\omega^{b}}{\tau^{m}}\right)\left[R_{i}^{e q}\left(\rho^{r}, \boldsymbol{u}\right)-R_{i}\right]+ \\
& +\frac{\omega^{r}}{\tau^{r}} \rho^{r} \frac{D}{b_{m} c^{2}}\left(\boldsymbol{u}^{r}-\boldsymbol{u}\right) \cdot \boldsymbol{c}_{i}+\frac{\omega^{b}}{\tau^{b}} \rho^{r} \frac{D}{b_{m} c^{2}}\left(\boldsymbol{u}^{b}-\boldsymbol{u}\right) \cdot \boldsymbol{c}_{i}+\mathcal{O}\left(u^{2}\right) .
\end{aligned}
$$

Writting lattice-Boltzmann equation, Eq. (4), in continuous variables, using a time scale $\delta$ and the length scale $h$ and using dimensionless variables $t^{*}=t / t_{c}$, where $t_{c}$ is a macroscopic time, $\boldsymbol{x}^{*}=\boldsymbol{x} / L$,

$$
\begin{aligned}
& \frac{\epsilon}{\mathcal{K}_{n}} \partial_{t^{*}} R_{i}+c_{i \alpha} \partial_{\alpha^{*}} R_{i}+\frac{1}{2} \frac{\epsilon^{2}}{\mathcal{K}_{n}} \partial_{t^{*} t^{*}} R_{i}+\frac{1}{2} \mathcal{K}_{n} c_{i \alpha} c_{i \beta} \partial_{\alpha^{*} \beta^{*}} R_{i}+ \\
&+\epsilon c_{i \alpha} \partial_{t^{*}} \partial_{\alpha^{*}} R_{i}=\frac{1}{\mathcal{K}_{n}}\left(\frac{\omega^{r}}{\tau^{r}}+\frac{\omega^{b}}{\tau^{m}}\right)\left[R_{i}^{e q}\left(\rho^{r}, \boldsymbol{u}\right)-R_{i}\right]+ \\
&+ \frac{\omega^{r}}{\tau^{r}} \frac{D}{b_{m} c^{2}} j^{r, 1} \cdot \boldsymbol{c}_{i}+\frac{\omega^{r}}{\tau^{m}} \frac{\rho^{r}}{\rho^{b}} \frac{D}{b_{m} c^{2}} j^{b, 1} \cdot \boldsymbol{c}_{i}
\end{aligned}
$$

where $\epsilon=\delta / t_{c}$ and

$$
j^{k, 1}=\sum_{i} K_{i}^{1} \boldsymbol{c}_{i}
$$

is the $\mathcal{K}_{n}$ first order contribution to species $K$ diffusive flux

$$
\boldsymbol{j}^{k}=\rho^{k}\left(\boldsymbol{u}^{k}-\boldsymbol{u}\right)=\mathcal{K}_{n} \boldsymbol{j}^{k, 1}+\mathcal{K}_{n}^{2} \boldsymbol{j}^{k, 2}+\ldots
$$

In Equation (18), the first term in the second member will be dominant when $\epsilon \sim \mathcal{K}_{n} \ll 1$ and the solution of

$$
\left(\frac{\omega^{r}}{\tau^{r}}+\frac{\omega^{b}}{\tau^{m}}\right)\left[R_{i}^{e q}\left(\rho^{r}, \boldsymbol{u}\right)-R_{i}\right]=0,
$$

gives the zero-th order solution to $R_{i}$, 


$$
R_{i}^{0}=R_{i}^{e q}\left(\rho^{r}, \boldsymbol{u}\right)
$$

Using the same reasoning for species $b$,

$$
B_{i}^{0}=B_{i}^{e q}\left(\rho^{b}, \boldsymbol{u}\right) .
$$

In the first order, $R_{i}^{1}$ is the solution of

$$
\begin{gathered}
\frac{\epsilon}{\mathcal{K}_{n}} \partial_{0^{*}} R_{i}+c_{i \alpha} \partial_{\alpha^{*}} R_{i}= \\
\left(\frac{\omega^{r}}{\tau^{r}}+\frac{\omega^{b}}{\tau^{m}}\right) R_{i}^{1}+\frac{\omega^{r}}{\tau^{r}} \frac{D}{b_{m} c^{2}} j^{r, 1} \cdot \boldsymbol{c}_{i}+\frac{\omega^{r}}{\tau^{m}} \frac{\rho^{r}}{\rho^{b}} \frac{D}{b_{m} c^{2}} j^{b, 1} \cdot \boldsymbol{c}_{i},
\end{gathered}
$$

giving,

$$
\begin{aligned}
& R_{i}^{1}=-\frac{\tau^{r} \tau^{m}}{\tau^{r} \omega^{b}+\tau^{m} \omega^{r}}\left\{-\omega^{r} \frac{D}{b_{m} c^{2}} c_{i \alpha} j_{\alpha}^{r, 1}\left(\frac{1}{\tau^{r}}-\frac{1}{\tau^{m}}\right)+\right. \\
& +\frac{1}{b}\left[-\partial_{\alpha}\left(\rho^{r} u_{\alpha}\right)+\omega^{r} u_{\alpha} \partial_{\alpha} \rho+\left(\partial_{\alpha} \rho^{r}-\omega^{r} \partial_{\alpha} \rho\right) c_{i \alpha}\right]+ \\
& \left.+\left[-\frac{D+2}{c^{2}} \omega^{r} u_{\beta} \partial_{\alpha} \rho+\frac{b D}{b_{m} c^{2}}+\frac{b D}{b_{m} c^{2}}\left(\partial_{\alpha}\left(\rho^{r} u_{\beta}\right)+u_{\beta} \partial_{\alpha} \rho^{r}\right)\right] c_{i \alpha} c_{i \beta},\right\} .
\end{aligned}
$$

A similar expression can be obtained for $B_{i}^{1}$.

\section{Macroscopic Equations in the Continuum Limit}

Macroscopic equations were found by replacing the distributions $R_{i}^{1}$ and $B_{i}^{1}$ in the $\mathcal{K}_{n}$ expansion of Eq.(4). Mass conservation equation for the $k$-species is obtained by multipying the resulting equation by 1 in the $\boldsymbol{c}_{i}$-space and by adding the $\mathcal{K}_{n}$ zero and first order contributions. At first order in $\mathcal{K}_{n}$, species- $r$ mass conservation equation was recovered in the following form,

$$
\partial_{t} \rho^{r}+\partial\left(\rho^{r} u_{\alpha}\right)+\partial_{\alpha}\left(j_{\alpha}^{r}\right)=0,
$$

where the r-species diffusive flux is given by

$$
j_{\alpha}^{r}=-\rho \mathcal{D} \partial_{\alpha}\left(\omega^{r}\right)=-\rho\left(\tau^{m}-1 / 2\right) \frac{b_{m} c^{2}}{b D} \partial_{\alpha}\left(\omega^{r}\right),
$$

in lattice units.

In this way, bynary diffusivity depends, only, on the cross relaxation time and does not depend on the mass fraction of the $r$ and $b$ species. This result is consistent with Chapman-Enskog resuts, considering the first order $\boldsymbol{c}$-moments of Boltzmann continuous equation, when the mass $m_{r}=m_{b}$ [7]. In fact, this restriction cancels the species diffusive flux dependence on pressure gradients. 
For the momentum equation, the lacking of energy conservation in present model restricts the analysis to the low-velocity, incompressible limit. In this case, after dropping-out the terms related to $\nabla \cdot \boldsymbol{u}, \mathcal{O}\left(u^{2}\right)$ and $\mathcal{O}(\boldsymbol{j} \cdot \boldsymbol{u})$, ChapmanEnskog analysis leads to

$$
\partial_{t}\left(\rho u_{\beta}\right)+\partial_{\alpha}\left(P \delta_{\alpha \beta}+\rho u_{\alpha} u_{\beta}\right)=\eta \partial_{\alpha}\left[\rho\left(\partial_{\alpha} u_{\beta}+\partial_{\beta} u_{\alpha}\right)\right],
$$

where $P=\rho c_{s}^{2}$ and the kinematic viscosity coefficient $\eta$, in lattice units, is given by

$$
\eta=\frac{c^{2}}{D+2}\left[\frac{A+B}{2\left(\rho^{r} \tau^{m}+\rho^{b} \tau^{r}\right)\left(\rho^{b} \tau^{m}+\rho^{r} \tau^{b}\right)}\right],
$$

where $A$ and $B$ are shown below,

$$
\begin{aligned}
& A=-\rho^{r} \rho^{b}\left(\tau^{m}\right)^{2}-\left(\rho^{r}\right)^{2} \tau^{b} \tau^{m}-\left(\rho^{b}\right)^{2} \tau^{r} \tau^{m}-\rho^{r} \rho^{b} \tau^{r} \tau^{b} \\
& B=2 \rho^{r} \rho^{b}\left(\tau^{m}\right)^{2}\left(\tau^{r}+\tau^{b}\right)+2 \tau^{r} \tau^{b} \tau^{m}\left[\left(\rho^{r}\right)^{2}+\left(\rho^{b}\right)^{2}\right]
\end{aligned}
$$

In this way, in the incompressible limit, Navier-Stokes equation is correctly retrieved in this LB approximation. Mixture viscosity appears as a function of the three parameters, $\tau^{r}, \tau^{b}, \tau^{m}$ and the mass fractions, $\rho^{r}, \rho^{b}$. When $\rho^{b} \rightarrow 0$,

$$
\eta=\frac{c^{2}}{D+2}\left(\tau^{r}-\frac{1}{2}\right)
$$

which is the correct expression for the kinematic viscosity of pure component $r$.

\section{Comparison between Theoretical Predictions and Simulation Results}

Theoretical predictions Eqs.(27) and (29) were compared with simulation results. Simulated diffusivity values, shown in Table 1, were obtained using the transient method presented in Flekoy [8]. Simulation was performed using a $200 \mathrm{x} 100 \mathrm{x}$ 3, D3Q19 lattice, with a 10 particles/site for the particle density. Viscosity was obtained in a $400 \times 20 \times 3$ lattice from the velocity profiles of a plane Poiseuille flow $[9]$.

There is a very good agreement between theoretical predictions and simulation results even for markedely different relaxation times, although, as it was to be expected, large values of $\tau$ and/or large differences in viscosity can, substantially, increase the simulation running time.

\section{Conclusion}

Intended to be used in diffusion-problems with large concentration gradients, in presently proposed model, relaxation times are non-linear functions of the $r$ and 
Table 1. Comparison between theoretical and simulated results

\begin{tabular}{ccccccc}
\hline$\tau^{r}$ & $\tau^{b}$ & $\tau^{m}$ & $\mathcal{D}$ theoretical & $\mathcal{D}$ simulated & $\eta$ theoretical & $\eta$ simulated \\
\hline 0.51 & 0.51 & 0.51 & 0.003333 & 0.003339 & 0.0033333 & 0.003467 \\
\hline 0.51 & 0.8 & 0.9 & 0.133333 & 0.1335 & 0.083020 & 0.086198 \\
\hline 0.51 & 2.0 & 3 & 0.833333 & 0.833984 & 0.378632 & 0.371125 \\
\hline 1 & 1 & 1 & 0.166667 & 0.166669 & 0.166667 & 0.172615 \\
\hline 1 & 3 & 1.5 & 0.333333 & 0.331565 & 0.366667 & 0.370507 \\
\hline 3 & 6 & 1.5 & 0.333333 & 0.329481 & 0.566667 & 0.561238 \\
\hline 1 & 3 & 3 & 0.833333 & 0.81903 & 0.583333 & 0.559141 \\
\hline
\end{tabular}

$b$ mass fractions. Previously, the following three-parameter linear BGK model was used

$$
\Omega_{i}^{k}=\frac{K_{i}^{e q}\left(\rho^{k}, \boldsymbol{u}^{k}\right)}{\tau^{k}}+\frac{K_{i}^{e q}\left(\rho^{k}, \boldsymbol{u}\right)}{\tau^{m}},
$$

with the same features of the model given by Eqs (8), (9). In this way, it preserves mass and momentum in collisions and gives a consistent hydrodynamics. Nevertheless, the use of constant relaxation times leads to constant mixture viscosity and this appear to be, only, realistic when concentration gradients are small. In present notation, Luo and Gimaji's model [5] can be written as

$$
\Omega_{i}^{r}=\frac{R_{i}^{e q}\left(\rho^{r}, \boldsymbol{u}^{r}\right)-R_{i}}{\tau^{r}}+\frac{1}{\tau_{m}} \omega^{b} R_{i}^{e q}\left(\rho^{r}, \boldsymbol{u}^{r}\right)\left(\boldsymbol{c}_{i}-\boldsymbol{u}\right) \cdot\left(\boldsymbol{u}^{r}-\boldsymbol{u}^{b}\right) .
$$

Although the authors did not report their mixture viscosity, the use of a constant relaxation time for $r-r$ collisions appear to be unrealistic when concentration gradients are important. In addition, contribution of $r-b$ collisions to $R_{i}$ distribution is considered in Luo and Gimaji's model to be, only, proportional to the first-order non equilibrium term $\left(\boldsymbol{u}^{r}-\boldsymbol{u}^{b}\right)$. This difference should reveals to be unimportant, considering the low-velocity limitations of both models. Nevertheless, as commented in the Introduction of present paper, a more detailed comparative analysis is needed, focusing, specially, the computational performance.

Acknowledgements. Authors are greatly indebted with CAPES (Coordenação de Aperfeiçoamento do Pessoal do Ensino Superior), CNPq (Brazilian Council of Scientifical and Technological Development), ANP (Brazilian National Agency of Petroleum) and Finep (Fundação Nacional de Estudos e Pesquisas) for the financial support.

\section{References}

1. Sofonea, V., Sekerka R. F.: BGK models for difusion in isothermal binary fluid systems. Physica A 299 (2001) 494-520 
2. Shan, X., Chen, H.: Lattice Boltzmann model for simulating flows with multiple phases and components. Phys. Rev. E 47 (1993) 1815-1819

3. Martys, N. S., Chen, H.: Simulation of multicomponent fluids in complex threedimensional geometries by the lattice Boltzmann method. Phys. Rev. E 53 (1996) $743-750$

4. Xu, K.: BGK-based scheme for multicomponent flow calculations, J. Comput. Phys. 134 (1997) $122-133$

5. Luo, L. S., Girimaji, S. S.: Lattice Boltzmann model for binary mixtures Phys. Rev. E 66(3): art. no. 035301 (2002)

6. Rothman, D. H., Zaleski, S.: Lattice-gas cellular automata: simple models of complex hydrodynamics. Cambridge Univesity Press, United Kingdom (1997)

7. Philippi, P. C., Brun, R.: Kinetic modeling of polyatomic-gas mixtures. Physica A 105 (1981) 147-168

8. Flekkøy, E. G.: Lattice Bhatnagar-Gross-Krook models for miscible fluids Phys. Rev. E 47 (1993) 4247-4257

9. Kadanoff, L. P., McNamara, G. R., Zanetti, G.: A Poiseuille Viscometer for Lattice Gas Automata. Complex Systems 1 (1987) 791-803 